\title{
Burkitt's lymphoma presenting with rapidly and multi-organ involvement: A case presentation
}

\author{
Ilhami Berber ${ }^{1 \#}$, Mehmet Ali Erkurt ${ }^{1}$, Mustafa Koroglu ${ }^{1}$, Irfan Kuku ${ }^{1}$, Emin Kaya ${ }^{1}$, \\ Recep Bentli ${ }^{2}$ \\ ${ }^{1}$ Department of Hematology, School of Medicine, Inonu University, Malatya, Turkey; \\ \#Corresponding Author: drilhamiberber@hotmail.com \\ ${ }^{2}$ Departman of General Medicine, Inonu University, Malatya, Turkey
}

Received 26 March 2013; revised 27 April 2013; accepted 15 May 2013

Copyright (C) 2013 Ilhami Berber et al. This is an open access article distributed under the Creative Commons Attribution License, which permits unrestricted use, distribution, and reproduction in any medium, provided the original work is properly cited.

\begin{abstract}
Burkitt lymphoma is an aggressive lymphoma and its clinical, immunologic, chemical features are well-known. Burkitt lymphoma is in highgrade lymphomas and can spread very rapidly. Burkitt lymphoma has been difficult to be cured with conventional chemotherapy for adults. Early autologous bone marrow should be kept in mind in first remission BL. We presented a case of presenting with rapid and multi organ involvement with BL. A 55-year-old male patient responded to the conventional chemotherapy for short-term and died 9 months after diagnosis because of relapse of Burkitt lymphoma.
\end{abstract}

Keywords: Burkitt Lymphoma; Rapidly; Bone Marrow Transplantation

\section{INTRODUCTION}

Burkitt's lymphoma (BL), Denis Burkitt description in 1958 of an aggressive tumor of young children that was characterized by frequent jaw and abdominal involvement [1]. Three distinct clinical forms BL are recognized: endemic, sporadic and immune deficiency-associated. In 1964, it was shown that Epstein-Barr virus played a role in the etiology of endemic BL. BL is $2 \%$ percent in non-hodgkin lymphoma $[2,3]$.

\footnotetext{
*The authors declare that they have no competing interests.

This report reflects the opinion of the authors and does not represent the official position of any institution or sponsor. MAE was responsible for reviewing previous research, journal hand searching, and drafting the report. IK and EK were responsible for provision of published trial bibliographies, and preparing photographs. RB and MK contributed to the final draft of the manuscript and analysis of relevant data. IB was responsible for project coordination. All authors read and approved the final manuscript.
}

Starry-sky pattern and high Ki-67 index are characteristic findings in BL. Typical immunophenotype is $\operatorname{slg}(+)$, CD10(+), CD20(+), Tdt(-), Ki67+(\%100), bcl2(-), bcl6(+). BL is associated with a translocation between the long arm of chromosome 8 , the site of the c-MYC oncogene $(8 \mathrm{q}, 24)$ and one of three locations on Ig genes $(8,14),(2,8),(8,22)$. BL is in high-grade lymphomas and can spread very rapidly, with tumors even doubling in size in less than 24 hours, it is human's most aggressive tumor. The endemic form presents as a jaw or facial bone tumor. The primary tumor can spread to extranodal sites (mesentery, ovary, testis, kidney, breast, and meninges). Bone marrow involvement is seen in less than $10 \%$. Involvement at the time of initial presentation, is a common complication of recurrent or resistant disease treatment. BL has been difficult to be cured with conventional chemotherapy for adults. More than half of those with Burkitt lymphoma can be cured with intensive chemotherapy. The cure rate may be lower if the cancer spreads to the bone marrow or spinal fluid. The outlook is poor if the cancer comes back after a remission [4-6]. We presented a case of presenting with rapidly and multi organ involvement with BL.

\section{CASE REPORT}

A 55-year-old male patient was diagnosed with Burkitt's lymphoma one year ago by another medical center. 8 cycles of Hyper-CVAD (course A: cyclophosphamide, vincristine, doxorubicin (also known as adriamycin), and dexamethasone, course B: consists of methotrexate and cytarabine) treatment was given. The patient remained in remission for 3 months. He applied with complaints of fatigue, shortness of breath, generalized bone pain and headache. Physical examination; pulse rate 112/min, respiratory rate $32 / \mathrm{min}$ and fever were $38.4^{\circ} \mathrm{C}$. The masses were detected on the back $(3 \times 2 \mathrm{~cm})$ (Figure 1) 


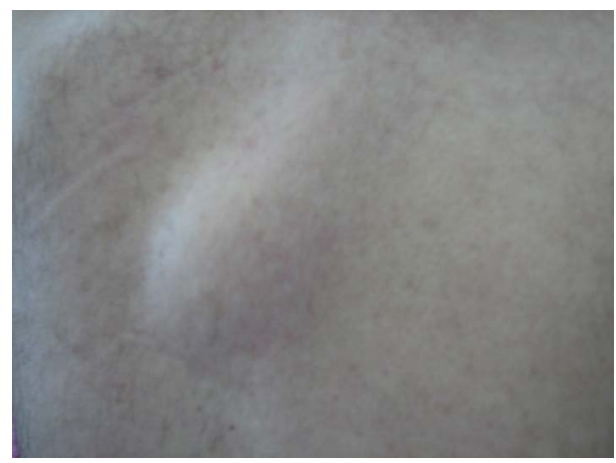

Figure 1. Back in themidline $5 \times 4 \mathrm{~cm}$ in diameter, adherent to the skin of thoracic vertebrae $6-7$ the level observed in a solid mass.

and in the scrotum $(3 \times 3 \mathrm{~cm})$.

Laboratory data were detected as leukocyte count 3200/microL, hemoglobine $8.2 \mathrm{~g} / \mathrm{dl}$, platelet count 5000/ microL, lactate dehydrogenase $790 \mathrm{u} / \mathrm{L}$ and other biochemical parameters were normal. Peripheral blood smear neutrophil $60 \%$, lymphocyte $35 \%$, monocyte $3 \%$, eucynophile $1 \%$, basophile $1 \%$, normochromic normocytic anemia. In brain magnetic resonance, approximately 5 $\mathrm{cm}$ mass in diameter was seen when located in the left temporal intra axial plant space occupying lesion with hemorrhagic areas (Figure 2). Lymphoma involvement was considered with this brain magnetic resonance. Involvement was not observed in bone marrow biopsy. Two numbers (as $1.5 \mathrm{~cm}$ and $1 \mathrm{~cm}$ ) nodular lesions were detected in the right lung by thoracic computed tomography (CT). Abdominal CT scan showed no pathology other than hepatomegaly. Body involvement was showed (bone intracranial, pelvic and skin and mediastinal) in positron emmission tomography (Figure 3). Burkitt's lymphoma cells on the back a mass of fine needle aspiration biopsy (Figure 4). Supportive therapy was given. Curative treatment could'not been able to start because of his poor performance. The patient's general condition did not improve and worsened. He died because of disease progression within a short time after recurrence.

\section{DISCUSSION}

New treatments increased disease free and overall survival rates in BL. Treatment of BL divides surgery, radiotherapy, chemotherapy and stem cell transplantation. In recent times BL was treated with CHOP (cyclophosphamide, hydroxydaunorubicin (also called doxorubicin or adriamycin), vincristine, prednisone or prednisolone) and Hyper-CVAD, then made between 1989-1996 NCI study, the more complex and more interest was the subject of an intensive treatment regimen. This so-called alternating cycles of CODOX-M/IVAC given cyclophosphamide, doxorubicin, vincristine and high-dose

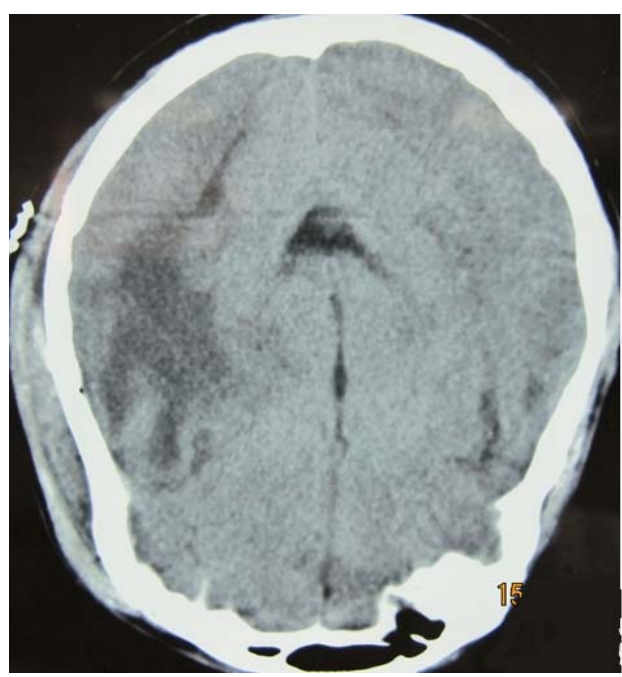

Figure 2. Brain MRI in 5-cm diameter mass in the left temporal area.

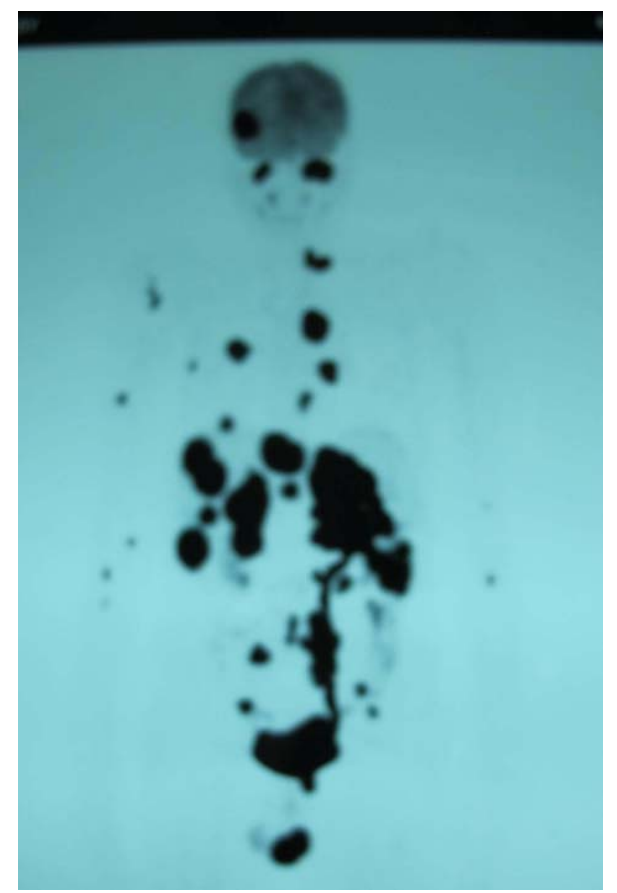

Figure 3. PET CT involvement is comonly observed.

methotrexate and ifosfamide, etoposide and consists of high-dose cytosine arabinoside $[5,6]$.

However, with currently-day regimens, disease free survival is in the $40 \%$ to $80 \%$ range for advanced $\mathrm{BL}$, obviating the need for early transplantation in most adult patients. Advanced-stage patients with central nervous system or marrow involvement previously had survival $<30 \%$ and were considered candidates for early transplantation [7]. Soussain C. et al. reports of salvage chemotherapy followed by autologous bone morrow transplantation have demonstrated varied results, with some 


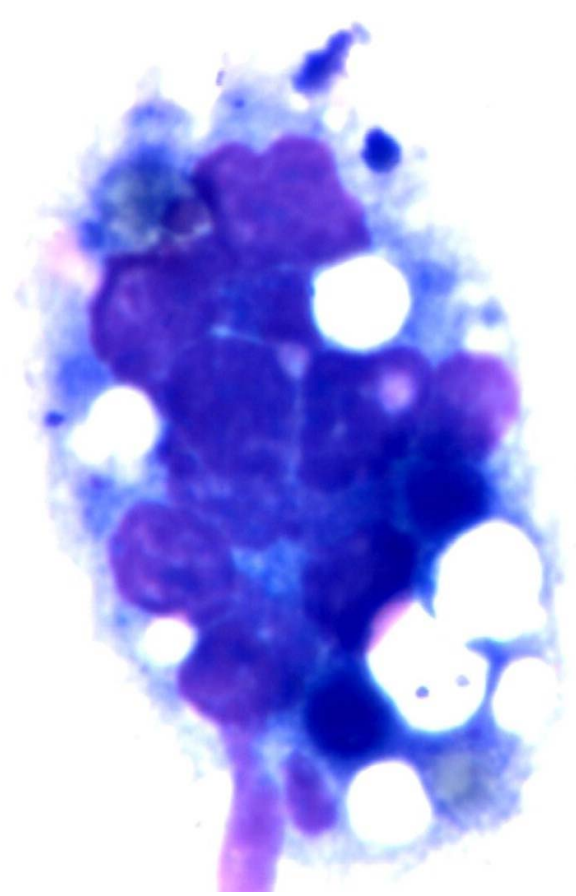

Figure 4. Skin fine-needle aspiration biopsy, dense core, narrow cytoplasm, large, basophili clymphoma cells stained.

studies reporting few long-term survivors [8]. Sweetenham et al. [9] transplanted 70 patients in first remission and 47 patients with relapsed disease. The 3-year overall survival rate was $72 \%$ for patients transplanted in first complet remission, $37 \%$ for patients in chemosensitive relapse, and $7 \%$ for patients with chemo-resistant relapse. Nademanee et al. [10] transplanted 10 patients with BL in first remission and reported 60\% 3-year disease free survival rate. Some centers advocate early autologous stem cell transplantation for patients with limited marrow involvement [11]. They also studied autologous bone morrow transplantation in refractory and relapsed disease and demonstrated 5-year disease free survival rate of $30 \%$ for patients transplanted in induction failure and $34 \%$ for relapsed patients [12]. Our patient admitted relaps BL. His clinic status was not suitable for autologous bone marrow transplantation. His disease worsened rapidly and he died within short time.

\section{CONCLUSION}

Early autologous bone marrow transplantation is promising and should be kept in mind in first remission BL.

\section{CONSENT}

Written informed consent was obtained from the patient's next of kin for publication of this manuscript and accompanying images. A copy of the written consent is available for review by the Editor-in-Chief of this journal.

\section{REFERENCES}

[1] Burkitt, D.P. (1983) The discovery of Burkitt's lymphoma. Cancer, 51, 1777-1786. doi:10.1002/1097-0142(19830515)51:10<1777::AID-CN CR2820511003>3.0.CO;2-E

[2] Armitage, J.O. and Weisenburger, D.D. (1998) New approach to classifying non-Hodgkin's lymphomas: Clinical features of the major histologic subtypes. Non-Hodgkin's Lymphoma Classification Project. Journal of Clinical Oncology, 16, 2780-2795.

[3] Jemal, A., Siegel, R., Ward, E., Hao, Y., Xu, J., Murray, T., et al. (2008) Cancer statistics. Cancer Statistics, 58, 7196. doi:10.3322/CA.2007.0010

[4] Anderson, J.R., Jenkin, R.D., Wilson, J.F., Kjeldsberg, C.R., Sposto, R., Chilcote, R.R., et al. (1993) Long-term follow-up of patients treated with COMP or LSA2L2 therapy for childhood non-Hodgkin's lymphoma: A report of CCG-551 from the Childrens Cancer Group. Journal of Clinical Oncology, 11, 1024-1032.

[5] Blum, K.A., Lozanski, G. and Byrd, J.C. (2004) Adult Burkitt leukemia and lymphoma. Blood, 104, 3009-3020. doi:10.1182/blood-2004-02-0405

[6] Evens, A.M. and Gordon, L.I. (2002) Burkitt's and Burkitt-like lymphoma. Current Treatment Options in Oncology, 3, 291-305. doi:10.1007/s11864-002-0029-9

[7] Fenaux, P., Bourhis, J.H. and Ribrag, V. (2001) Burkitt's acute lymphocytic leukemia (L3ALL) in adults. Hematology/Oncology Clinics of North America, 15, 37-50. doi:10.1016/S0889-8588(05)70199-4

[8] Soussain, C., Patte, C., Ostronoff, M., Delmer, A., RigalHuguet, F., Cambier, N., et al., (1995) Small noncleaved cell lymphoma and leukemia in adults. A retrospective study of 65 adults treated with the LMB pediatric protocols. Blood, 85, 664-674.

[9] Sweetenham, J.W., Pearce, R., Taghipour, G., Blaise, D., Gisselbrecht, C. and Goldstone, A.H. (1996) Adult Burkitt's and Burkitt-like non-Hodgkin's lymphoma-Outcome for patients treated with high-dose therapy and autologous stem-cell transplantation in first remission or at relapse: Results from the European Group for blood and marrow transplantation. Journal of Clinical Oncology, 14, 2465-2472.

[10] Nademanee, A., Molina, A., O’Donnell, M.R., Dagis, A., Snyder, D.S., Parker, P., et al. (1997) Results of high-dose therapy and autologous bone marrow/stem cell transplantation during remission in poor-risk intermediate- and high-grade lymphoma: International index high and highintermediate risk group. Blood, 90, 3844-3852.

[11] Van Imhoff, G.W., van der Holt, B., MacKenzie, M.A., Ossenkoppele, G.J., Wijermans, P.W., Kramer, M.H., et al., (2005) Short intensive sequential therapy followed by autologous stem cell transplantation in adult Burkitt, Burkitt-like and lymphoblastic lymphoma. Leukemia, 19, 945- 
952. doi:10.1038/sj.leu.2403733

[12] Nademanee, A., Molina, A., Dagis, A., Snyder, D.S., O’Donnell, M.R., Parker, P., et al., (2000) Autologous stem-cell transplantation for poor-risk and relapsed intermediate- and high-grade non-Hodgkin's lymphoma. Clinical Lymphoma, 1, 46-54. doi:10.3816/CLM.2000.n.004 